\title{
INFLUÊNCIA DO MÉTODO EXTRATIVO NO TEOR DE FLAVONOIDES DE Cnidoscolus quercifolius POHL (EUPHORBIACEAE) E ATIVIDADE ANTIOXIDANTE
}

Daniel da S. Torres ${ }^{a}$, Emanuella C. V. Pereira ${ }^{a}$, Pedrita A. Sampaio ${ }^{a}$, Nathália A. C. de Souza ${ }^{a}$, Christiane A. A. Ferraz ${ }^{b}$, Ana P. de Oliveira ${ }^{\mathrm{a}, \mathrm{b}}$, Celuane A. Moura ${ }^{\mathrm{b}}$, Jackson R. G. S. Almeida ${ }^{\mathrm{a}, \mathrm{b}}$, Pedro J. Rolim-Neto ${ }^{\mathrm{c}}$, Raimundo G. de Oliveira-Júnior ${ }^{\mathrm{a}, \mathrm{b}}$ e Larissa A. Rolima, a,**

${ }^{a}$ Central de Análise de Fármacos, Medicamentos e Alimentos, Universidade Federal do Vale do São Francisco, 56304-205 Petrolina - PE, Brasil

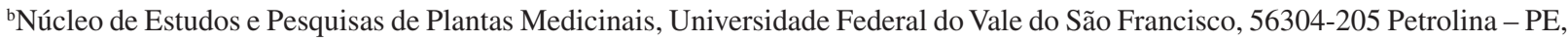
Brasil

'Laboratório de Tecnologia dos Medicamentos, Universidade Federal de Pernambuco, 50740-521 Recife - PE, Brasil

Recebido em 30/01/2018; aceito em 11/04/2018; publicado na web em 16/05/2018

INFLUENCE OF EXTRACTION PROCESS ON FLAVONOID CONTENT FROM Cnidoscolus quercifolius POHL (EUPHORBIACEAE) AND ANTIOXIDANT ACTIVITY. Cnidoscolus quercifolius Pohl is a Brazilian medicinal plant from Caatinga biome and possesses several pharmacological properties. Phytochemical investigations have attributed some of these properties to the presence of flavonoids. In this paper, we report the influence of the extraction process on the flavonoid content obtained from leaves of C. quercifolius and its antioxidant activity. A factorial design $2^{2}$ was performed, using temperature and agitation as independent variables. Qualitative and quantitative analyzes of the extracts were performed by HPLC-DAD. Rutin and apigenin were identified in the extracts and chosen as analytical markers. Pareto chart and Surface response plots suggested that rutin content was increased up to 3.93 times when temperature was employed during the extraction process. However, apigenin content was significantly reduced in the method whose temperature or agitation were used $(p<0.05)$. A higher antioxidant activity was observed in extracts whose rutin extraction was favored, suggesting that this flavonoid contributes at least in part to the antioxidant potential of the species.

Keywords: medicinal plants; flavonoids; factorial design; extraction conditions.

\section{INTRODUÇÃO}

Cnidoscolus quercifolius (syn. C. phyllacanthus (Mull. Arg.) Pax \& L. Hoffm.) é uma planta medicinal brasileira, endêmica do bioma Caatinga, frequentemente encontrada no semiárido nordestino, em especial nos estados de Pernambuco, Bahia, Ceará, Paraíba, Piauí e Rio Grande do Norte, além do estado de Minas Gerais, na região sudeste. Essa espécie é conhecida popularmente como "favela", "faveleira" ou "urtiga-branca", sendo tradicionalmente utilizada no tratamento de dores e processos inflamatórios, infecções, problemas estomacais e cicatrização de feridas. ${ }^{1,2}$

Estudos farmacológicos realizados previamente demonstraram que extratos etanólicos obtidos das folhas e cascas do caule da espécie apresentam atividade antinociceptiva ${ }^{3}$ e anti-inflamatória ${ }^{4}$ em modelos animais. Além disso, também foi demonstrado o potencial antioxidante e antimicrobiano de diferentes extratos de $C$. quercifolius..$^{5-7}$ Essas propriedades terapêuticas têm sido frequentemente correlacionadas ao elevado teor de compostos fenólicos e, principalmente, flavonoides.

Recentemente, Paula et al. ${ }^{8}$ descreveu o isolamento de triterpenos e bis-norditerpenos das cascas do caule da planta. De fato, bis-norditerpenos do tipo benzilcicloheptano (denominados de favelinhas $)^{9,10}$ e novos derivados ciclopropano (favelanona) e ciclobuteno tetracíclicos (neofavelanona) ${ }^{11}$ têm sido relatados com frequência para essa espécie, podendo ser considerados seus marcadores quimiotaxonômicos. Esses compostos apresentam atividade citotóxica significativa em diversas linhagens de células tumorais humanas, sendo atribuída a eles a propriedade antitumoral verificada para extratos de C. quercifolius. ${ }^{8,12}$
Apesar de C. quercifolius ser considerada uma espécie de uso tradicional amplamente difundido em diversas comunidades do Nordeste brasileiro, com algumas de suas propriedades farmacológicas avaliadas e comprovadas cientificamente, ainda existem poucos estudos químicos e farmacológicos envolvendo esta planta. Além disso, as atividades farmacológicas verificadas para C. quercifolius são normalmente atribuídas a classes de metabólitos secundários distintas, o que torna necessário o desenvolvimento de métodos extrativos que favoreçam a obtenção de compostos de interesse para determinadas propriedades terapêuticas. Nesse trabalho, nós relatamos a influência do método extrativo no teor de flavonoides obtidos das folhas de $C$. quercifolius, em especial os flavonoides rutina e apigenina, que puderam ser identificados através de análises por CLAE-DAD. Adicionalmente, foi avaliada a atividade antioxidante in vitro dos extratos obtidos, correlacionando-a com o teor de flavonoides verificado.

\section{PARTE EXPERIMENTAL}

\section{Material vegetal}

As folhas da espécie $C$. quercifolius foram coletadas no Campus de Ciências Agrárias (CCA), da Universidade Federal do Vale do São Francisco (UNIVASF), no município de Petrolina-PE, em agosto de 2015, e foram comparadas com uma exsicata da planta previamente depositada no Herbário do Vale do São Francisco ( ${ }^{\circ}$ 19202), na UNIVASF. Em seguida, o material $(10 \mathrm{~kg})$ foi seco em estufa de circulação de ar e pulverizadas em moinho de facas, fornecendo 2,044 kg da planta pulverizada. 


\section{Aplicação de planejamento fatorial $2^{2}$ para avaliação do} método extrativo

Para avaliar a influência do método de extração sobre o teor dos flavonoides obtidos da espécie, foi realizado um planejamento fatorial envolvendo dois fatores estudados em dois níveis diferentes (+1 e -1), caracterizando um planejamento fatorial $2^{2}$. Para todos os métodos, foi utilizada a proporção planta/solvente de $1: 10(\mathrm{~m} / \mathrm{v})$, utilizando $10 \mathrm{~g}$ da planta pulberizada para $100 \mathrm{~mL}$ de solvente. O tempo de extração foi mantido em duas horas e todos os extratos foram preparados utilizando etanol absoluto como solvente. Os extratos foram preparados em triplicata, variando-se a temperatura de extração (a frio ou a quente) e a agitação do meio (com ou sem agitação), conforme descrito na Tabela 1. Em seguida, a solução extrativa obtida foi levada ao aparelho evaporador rotativo para eliminação do solvente, resultando nos diferentes extratos brutos ( $\mathrm{Cq} 1, \mathrm{Cq} 2, \mathrm{Cq} 3$ e $\mathrm{Cq} 4)$. O rendimento do método extrativo foi calculado com base na massa final obtida para cada extrato em relação à massa de material vegetal utilizada, sendo expresso em percentual $(\%, \mathrm{~m} / \mathrm{m})$.

Tabela 1. Variáveis independentes (temperatura e agitação) e níveis (-1 e +1) do planejamento fatorial utilizado com a espécie C. quercifolius

\begin{tabular}{cccc}
\hline $\begin{array}{c}\text { Método } \\
\text { extrativo }\end{array}$ & Extrato & $\begin{array}{c}\text { Temperatura } \\
\left({ }^{\circ} \mathrm{C}\right)\end{array}$ & $\begin{array}{c}\text { Agitação } \\
(\mathrm{rpm})\end{array}$ \\
\hline Método 1 & $\mathrm{Cq} 1$ & $25(-1)$ & $0(-1)$ \\
Método 2 & $\mathrm{Cq} 2$ & $45(+1)$ & $0(-1)$ \\
Método 3 & $\mathrm{Cq} 3$ & $25(-1)$ & $1000(+1)$ \\
Método 4 & $\mathrm{Cq} 4$ & $45(+1)$ & $1000(+1)$ \\
\hline
\end{tabular}

\section{Análises por CLAE-DAD}

Todos os extratos foram submetidos a uma análise quali-quantitativa por CLAE-DAD visando a identificação e quantificação dos flavonoides presentes na espécie. As análises foram realizadas em cromatógrafo líquido Shimadzu ${ }^{\circledR}$ (LC-20 AT) equipado com um amostrador automático (SIL-20 A) e um detector de arranjo de diodos (SPD-M20A) controlado por software LC-Solution 1.0; e uma coluna Zorbax, Agilent ( 250 x 4,6 mm e 5 um) com pré-coluna. Todas as soluções foram degaseificadas e filtradas através de membrana filtrante de 0,22 $\mu \mathrm{m}$ (Chromafil® Xtra, EUA), antes de serem analisadas no cromatógrafo. Os extratos (Cq1-Cq4) foram analisados na concentração de $10 \mathrm{mg} / \mathrm{mL}$, solubilizados em metanol, à temperatura de $37^{\circ} \mathrm{C}$, com fluxo de $1 \mathrm{~mL} \mathrm{~min}{ }^{-1}$ e volume de injeção de $50 \mu \mathrm{L}$, sendo o tempo de análise de 70 min e a fase móvel constituída de dois solventes: solvente A (solução de ácido fórmico $0,1 \%$ ) e solvente B (acetonitrila); em eluição gradiente: 0 min (100\% A), 50 min (40\% A e 60\% B), 60 min (40\% A e 60\% B), $70 \mathrm{~min}(100 \%$ A). Todas as análises foram realizadas em triplicata e os cromatogramas foram obtidos em $320 \mathrm{~nm}$.

Para identificação dos compostos, foram utilizados padrões analíticos obtidos da Sigma ${ }^{\circledR}$ (Brasil). Todos os padrões foram analisados por CLAE-DAD sob as mesmas condições de análise dos extratos. A identificação dos compostos foi realizada com base na comparação dos valores de tempo de retenção e dos comprimentos de onda de absorção máxima ( $\lambda \max )$ dos picos encontrados nos cromatogramas das amostras com aqueles verificados nos cromatogramas dos padrões analíticos.

\section{Quantificação dos flavonoides rutina e apigenina}

Após o desenvolvimento dos métodos extrativos e a identificação dos compostos, foi realizada a curva padrão para quantificação dos flavonoides rutina (CAS 153-18-4, Sigma ${ }^{\circledR}$ ) e apigenina (CAS 52036-5, Sigma ${ }^{\circledR}$ ) por CLAE-DAD. A quantificação desses marcadores nos diferentes extratos foi realizada a partir da equação da reta obtida de suas respectivas curvas de calibração: 40 a $60 \mu \mathrm{g} \mathrm{mL}^{-1}$ de rutina $\left(\mathrm{y}=32588 \mathrm{x}-294690\right.$, com $\left.\mathrm{R}^{2}=0,98\right)$ e 0 a $1 \mu \mathrm{g} \mathrm{mL} \mathrm{L}^{-1}$ de apigenina $\left(y=6000000 x-187553\right.$, com $\left.R^{2}=0,99\right)$. O teor dos flavonoides foi expresso em $\mu \mathrm{g} / \mathrm{mg}$ de extrato, empregando-se valores de média \pm desvio padrão.

\section{Avaliação da atividade antioxidante}

A atividade antioxidante dos extratos de $C$. quercifolius foi avaliada através do método do sequestro do radical 2,2-difenil-1-picrilhidrazila (DPPH). ${ }^{13,14}$ Soluções estoques das amostras (Cq1-Cq4, $1 \mathrm{mg} / \mathrm{mL}$ ) e padrão (ácido ascórbico, $1 \mathrm{mg} / \mathrm{mL}$ ) foram preparadas e diluídas até concentrações finais de 1, 3, 9, 27, 81 e $243 \mu \mathrm{g} / \mathrm{mL}$, em etanol absoluto. Uma solução contendo o radical DPPH foi preparada em etanol, na concentração de $50 \mu \mathrm{g} \mathrm{mL}^{-1}$. Em seguida, adicionou-se $1 \mathrm{~mL}$ desta solução a $2,5 \mathrm{~mL}$ de cada uma das soluções diluídas dos extratos. Após 30 minutos de reação em temperatura ambiente e ao abrigo da luz, foram realizadas as leituras das absorbâncias em espectrofotômetro (QUIMIS ${ }^{\circledR}$, Brasil), em 518 nm. Os valores de absorbância foram convertidos em percentual de atividade antioxidante através da seguinte equação: $\mathrm{AA}(\%)=\left[\left(\mathrm{A}_{\text {controle }}-\mathrm{A}_{\text {amostra }}\right) /\right.$ $\left.\mathrm{A}_{\text {controle }}\right] \times 100$, em que $\mathrm{A}_{\text {controle }}$ representa a absorbância do controle negativo ( $1 \mathrm{~mL}$ da solução de DPPH $+2,5 \mathrm{~mL}$ de etanol absoluto) e $A_{\text {amostra }}$ representa a absorbância da amostra. Soluções de etanol $(1 \mathrm{~mL})$ com as amostras $(2,5 \mathrm{~mL})$ foram utilizadas como branco. Todos os experimentos foram realizados em triplicata e os valores de percentual de atividade antioxidante foram convertidos para $\mathrm{CE}_{50}$ através de análise de regressão não linear.

\section{Análise estatística}

Todos as determinações foram realizadas em triplicata. A análise dos dados da quantificação dos flavonoides apigenina e rutina foi realizada utilizando o software OriginPro $8^{\circledR}$. Os gráficos de Pareto e de Superfície-Resposta foram obtidos através do software Statistica ${ }^{\circledR}$ (StatSoft, versão 5.0), considerando os valores significativamente diferentes quando $p<0,05$. Os valores de $\mathrm{CE}_{50}$ e de percentual de atividade antioxidante foram calculados com o auxílio do programa GraphPad Prism6 ${ }^{\circledast}$.

\section{RESULTADOS E DISCUSSÃO}

Inicialmente, foi avaliado o rendimento dos extratos obtidos de acordo com as condições de extração utilizadas. De modo geral, o emprego de temperatura e agitação facilitou a extração dos constituintes químicos da planta (Tabela 2), um resultado previsível, uma vez que esses fatores aumentam a difusão e solubilidade do composto, além de diminuírem a viscosidade do solvente, resultando assim na melhoria da extração.

Após obtenção, todos os extratos foram analisados por CLAEDAD sob as mesmas condições cromatográficas. Todos os extratos apresentaram perfil cromatográfico semelhante, variando apenas a intensidade dos picos registrados (Figura 1). Os cromatogramas obtidos $(320 \mathrm{~nm})$ revelaram diversos picos com bandas de absorção UV características de derivados flavonoídicos ( ${ }_{\max }$ 250-270 e 320-360 $\mathrm{nm})$. Dentre esses, dois foram identificados como sendo os flavonoides rutina e apigenina, ${ }^{15}$ com base na comparação do tempo de retenção (TR) e o perfil de absorção UV registrados para os padrões analíticos utilizados (Figura 2). A Tabela 2 sumariza os valores de TR e max , além do teor de rutina e apigenina calculados para cada extrato. 


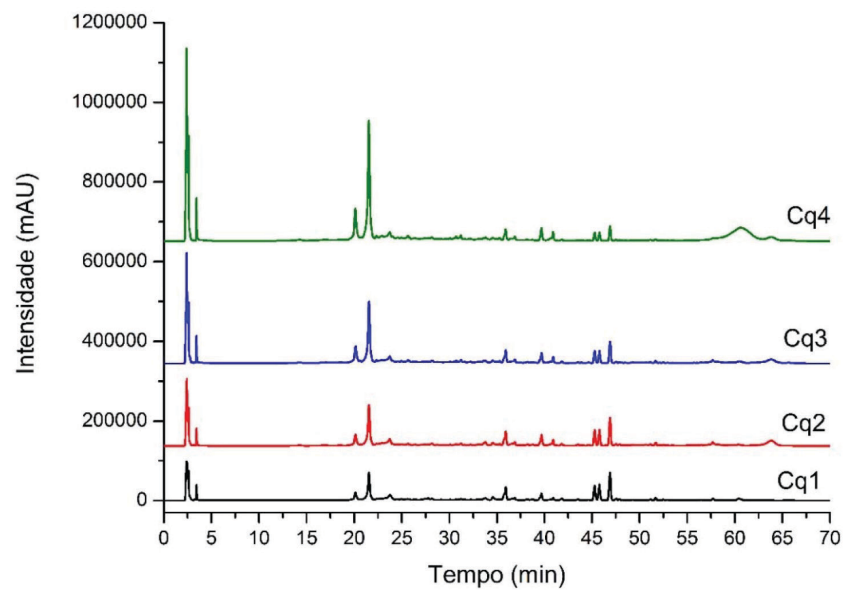

Figura 1. CLAE-DAD (320 nm) dos extratos (Cq1-Cq4) obtidos através diferentes métodos extrativos empregados, conforme apresentado na Tabela 1

A aplicação do planejamento fatorial permitiu avaliar a influência dos fatores temperatura e agitação na extração dos flavonoides identificados. O gráfico de Superfície-Resposta apresentado na Figura 3 demonstra que quanto maior a temperatura empregada, maior o teor de rutina obtido durante a preparação dos extratos. O diagrama de Pareto corrobora essa informação, revelando que a temperatura influencia positivamente na extração da rutina $(p<0.05)$ (Figura 3 ).
Por outro lado, o emprego de agitação ou a combinação das duas variáveis (agitação + temperatura) não interferem de modo significativo no teor do composto. Desse modo, $\mathrm{Cq} 3$ e $\mathrm{Cq} 4$ foram consideradas as melhores condições de extração da rutina, apresentando teores de

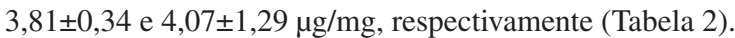

$\mathrm{O}$ uso da temperatura como um fator que melhora a extração de compostos fenólicos, em especial os flavonoides, tem sido amplamente descrito na literatura. ${ }^{16-18}$ Além disso, por se tratar de um derivado glicosilado, é natural que a rutina seja mais facilmente extraída em temperaturas elevadas. ${ }^{19}$ Contudo, também é sabido que o emprego de altas temperaturas pode levar à degradação desse composto, ${ }^{20,21}$ o que torna necessário o desenvolvimento de novas metodologias de otimização do método extrativo a fim de selecionar as melhores condições de extração, sem que a substância seja degradada durante o processo extrativo.

Diferentemente do que foi verificado para rutina, o gráfico de Superfície-Resposta apresentado na figura 4 revela que o uso de temperatura ou agitação diminuem a eficiência de extração do flavonoide apigenina. De acordo com o diagrama de Pareto (figura 4), o emprego dessas variáveis influencia negativamente na extração do composto $(p<0.05)$, justificando o menor teor de apigenina verificado para o extrato $\mathrm{Cq} 4\left(0,18 \pm 0,02 \mu \mathrm{g} \mathrm{mg}^{-1}\right)$ e o maior teor verificado para a condição $\operatorname{Cq} 1\left(0,26 \pm 0,01 \mu \mathrm{g} \mathrm{mg}^{-1}\right)$.

Levando em consideração que os flavonoides têm sido relatados como os constituintes químicos responsáveis pelo potencial antioxidante de $C$. quercifolius, ${ }^{5-7}$ nós resolvemos investigar a atividade
(A)

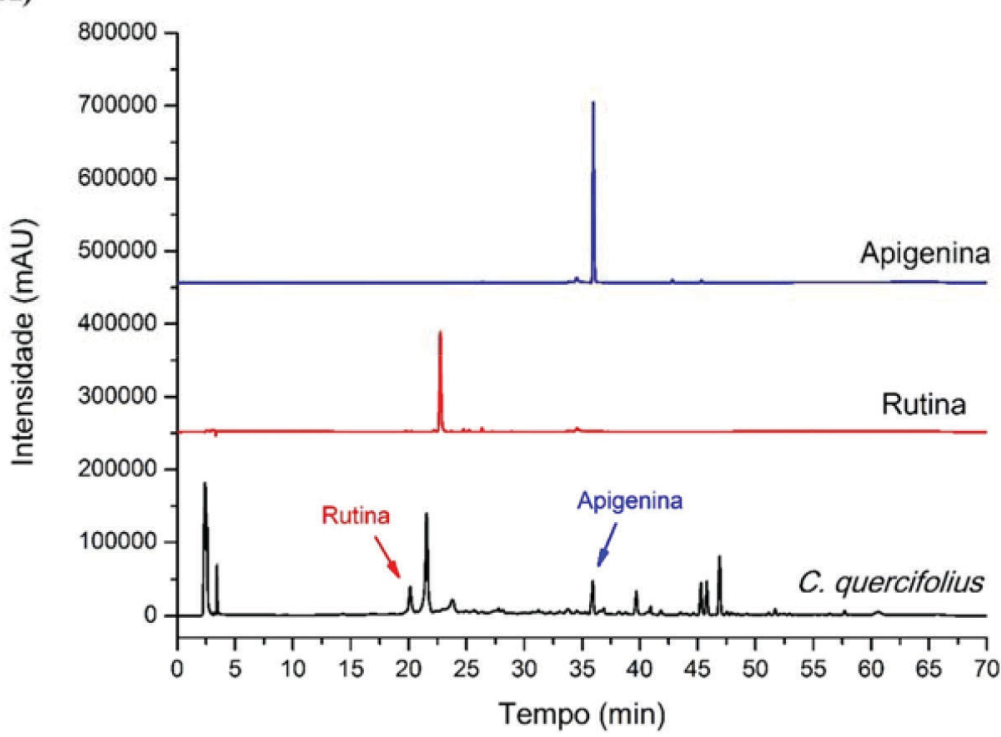

(B)

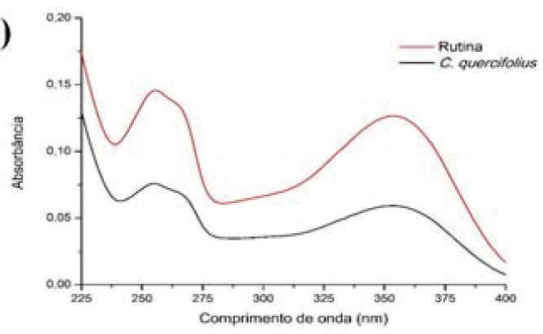

(C)

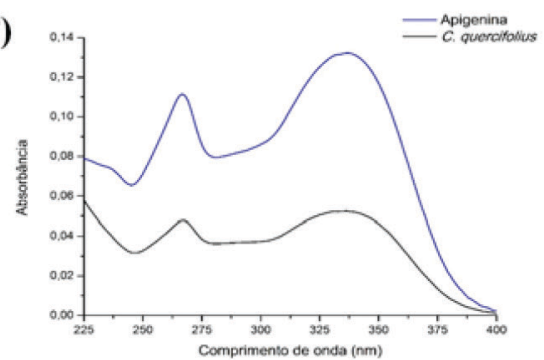

Figura 2. CLAE-DAD $(320 \mathrm{~nm})$ dos padrões rutina e apidenina, e do extrato de C. quercifolius (A). Espectro de absorção UV obtidos para rutina (B) e apigenina (C), comparados aos picos correspondentes em extratos de C. quercifolius

Tabela 2. Determinação do rendimento total (RT), tempo de retenção (TR) e comprimentos de onda de absorção máxima no espectro UV, além do teor verificado para os flavonoides rutina e apigenina em cada condição extrativa (Cq1-Cq4)

\begin{tabular}{|c|c|c|c|c|c|c|c|}
\hline \multirow[t]{2}{*}{ Extrato } & \multirow{2}{*}{$\begin{array}{c}\mathrm{RT} \\
(\%, \mathrm{~m} / \mathrm{m})\end{array}$} & \multicolumn{3}{|c|}{ Rutina } & \multicolumn{3}{|c|}{ Apigenina } \\
\hline & & $\begin{array}{c}\mathrm{TR} \\
(\mathrm{min})\end{array}$ & $\begin{array}{l}\lambda_{\max } \\
(\mathrm{nm})\end{array}$ & $\begin{array}{c}\text { Teor } \\
\left(\mu \mathrm{g} \mathrm{mg}^{-1}\right)\end{array}$ & $\begin{array}{c}\mathrm{TR} \\
(\mathrm{min})\end{array}$ & $\begin{array}{l}\lambda_{\max } \\
(\mathrm{nm})\end{array}$ & $\begin{array}{c}\text { Teor } \\
\left(\mu \mathrm{g} \mathrm{mg}^{-1}\right)\end{array}$ \\
\hline $\mathrm{Cq} 1$ & $3,71 \pm 0,87$ & 21,29 & 255,353 & $2,63 \pm 0,24$ & 35,82 & 267,337 & $0,26 \pm 0,01$ \\
\hline $\mathrm{Cq} 2$ & $8,24 \pm 0,82$ & 21,23 & 255,353 & $3,00 \pm 0,32$ & 35,87 & 267,337 & $0,23 \pm 0,01$ \\
\hline $\mathrm{Cq} 3$ & $3,93 \pm 0,99$ & 21,27 & 255,353 & $3,81 \pm 0,34$ & 35,87 & 267,337 & $0,25 \pm 0,01$ \\
\hline $\mathrm{Cq} 4$ & $10,31 \pm 0,97$ & 21,23 & 255,353 & $4,07 \pm 1,29$ & 35,85 & 267,337 & $0,18 \pm 0,02$ \\
\hline
\end{tabular}

Valores de RT e teor são expressos como média \pm desvio padrão. 
(A)

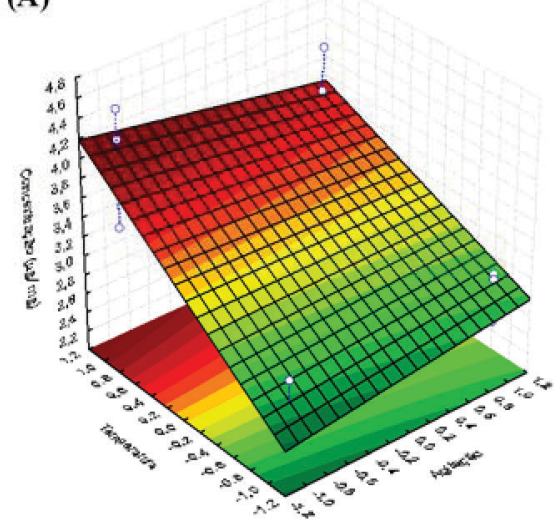

(B)

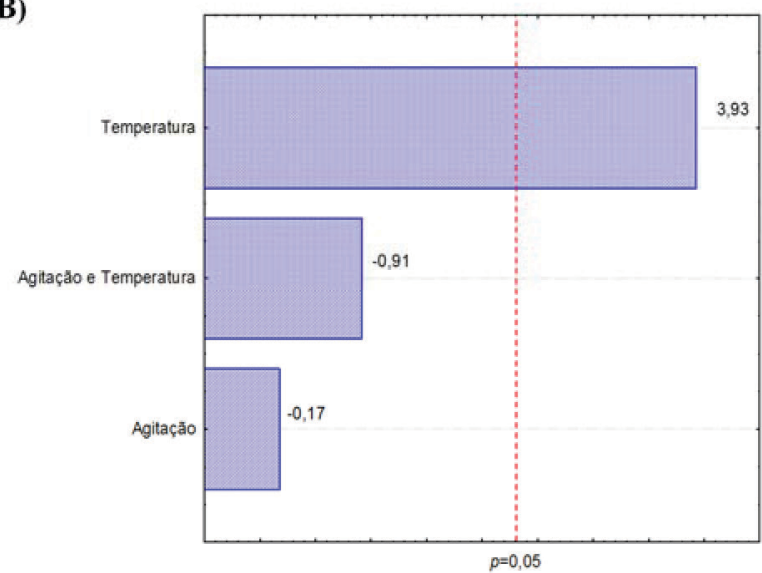

Figura 3. Gráfico de Superfície-Resposta (A) e diagrama de Pareto (B) mostrando a influência dos fatores temperatura e agitação no teor de rutina obtido durante o processo extrativo

(A)

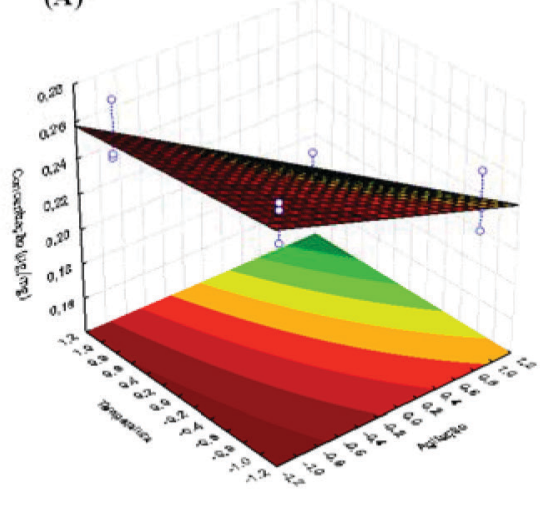

(B)

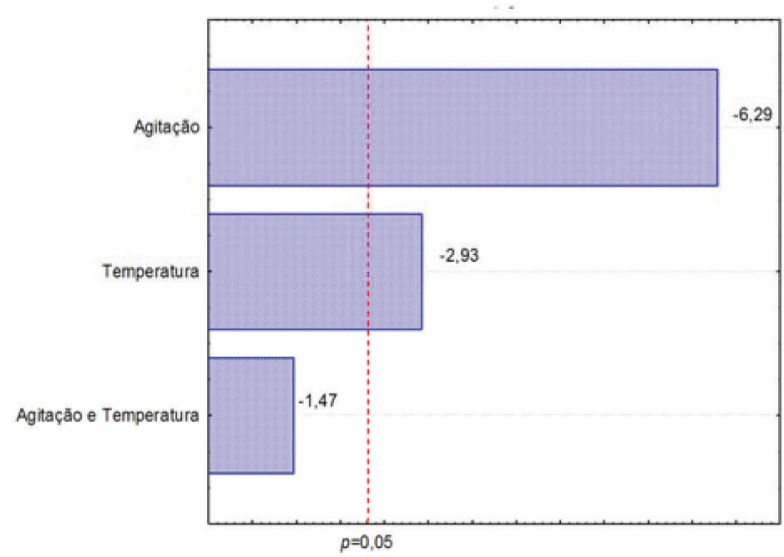

Figura 4. Gráfico de Superfície-Resposta (A) e diagrama de Pareto (B) mostrando a influência dos fatores temperatura e agitação no teor de apigenina obtido durante o processo extrativo

antioxidante dos extratos obtidos nas diferentes condições (Cq1-Cq4), utilizando o método do sequestro do radical DPPH. Este é um modelo clássico para avaliação do potencial antioxidante in vitro de amostras de natureza orgânica, sendo amplamente empregado na avaliação da atividade antioxidante de produtos naturais, incluindo extratos vegetais. ${ }^{13,14}$ De acordo com a Figura 5, foi verificado que o extrato Cq4 apresentou maior atividade antioxidante em comparação com os demais extratos, alcançando cerca de $90 \%$ de atividade na última concentração testada $\left(243 \mu \mathrm{g} \mathrm{mL} \mathrm{L}^{-1}\right)$. De fato, Cq4 apresentou o menor valor de $\mathrm{CE}_{50}\left(75,89 \pm 2,71 \mu \mathrm{g} \mathrm{mL} \mathrm{m}^{-1}\right)$ entre os extratos (Tabela 3), sugerindo que o emprego de fatores que favorecem a extração do flavonoide rutina tendem a melhorar a atividade antioxidante do extrato. Isso porque esse flavonoide apresenta elevado potencial antioxidante tanto em modelos in vitro, quanto em modelos in vivo, sendo utilizado, inclusive, como uma substância de referência (controle positivo) em alguns testes. ${ }^{22,23}$

\section{CONCLUSÃO}

O planejamento fatorial aplicado nesse estudo permitiu identificar a temperatura como sendo um fator que favorece a extração de rutina, mas desfavorece a extração de apigenina. A agitação também prejudica a extração de apigenina, mas não influencia significativamente no teor de rutina. Esses resultados sugerem que diferentes condições de temperatura e agitação podem ser empregadas na extração de flavonoides de C. quercifolius a depender do tipo de substâncias que

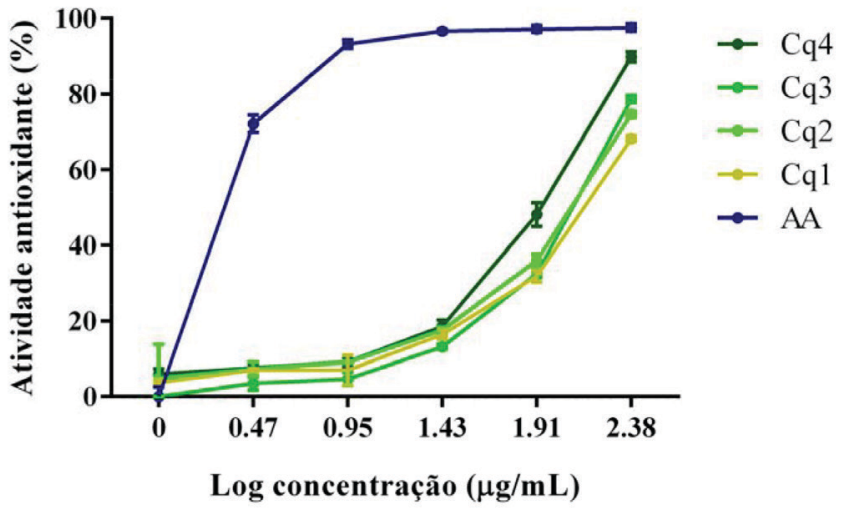

Figura 5. Atividade antioxidante (\%) verificada para os extratos obtidos em diferentes condições (Cq1-Cq4) e para o padrão ácido ascórbico (AA)

se deseja obter (flavonoides glicosilados, como rutina; ou agliconas, como apigenina). Além disso, foi verificado uma maior atividade antioxidante quando empregadas condições que favoreceram a extração de rutina, sugerindo que este flavonoide pode estar relacionado, pelo menos em parte, ao potencial antioxidante da espécie. Vale ressaltar que outros compostos fenólicos também podem ter contribuído para a melhoria da atividade antioxidante do extrato, sendo necessário o desenvolvimento de estudos fitoquímicos posteriores que permitam o isolamento e caracterização desses constituintes.

Embora preliminar, esse trabalho fornece informações 
Tabela 3. Valores de $\mathrm{CE}_{50}$ obtidos para os extratos (Cq1-Cq4) e para o padrão ácido ascórbico (AA) no teste do sequestro do radical DPPH

\begin{tabular}{cccccc}
\hline Amostra & AA & Cq1 & Cq2 & Cq3 & Cq4 \\
\hline $\mathrm{CE}_{50}\left(\mu \mathrm{g} \mathrm{mL}^{-1}\right)$ & $2,55 \pm 0,25$ & $135,6 \pm 5,52$ & $111,3 \pm 4,35$ & $116,2 \pm 3,38$ & $75,89 \pm 2,71$ \\
\hline
\end{tabular}

$\mathrm{CE}_{50}$ é definida como a concentração necessária para atingir $50 \%$ do efeito máximo. Os valores de $\mathrm{CE}_{50}$ foram calculados através de regressão não linear, e os resultados estão expressos como média \pm desvio padrão.

importantes para estudos posteriores que visem a otimização do processo extrativo de flavonoides de $C$. quercifolius, baseado nos compostos de interesse e nas atividades farmacológicas desejadas.

\section{AGRADECIMENTOS}

Os autores agradecem à FACEPE (Fundação de Amparo à Ciência e Tecnologia do Estado de Pernambuco), ao $\mathrm{CNPq}$ (Conselho Nacional de Desenvolvimento Científico e Tecnológico) e à UNIVASF (Universidade Federal do Vale do São Francisco) pelo apoio financeiro.

\section{REFERÊNCIAS}

1. Albuquerque, U. P.; Araujo, E. L.; El-Deir, A. C. A.; Lima, A. L. A.; Souto, A.; Bezerra, B. M.; Ferraz, E. M. N.; Freire, E. M. X.; Sampaio, E. V. S. B.; Las-Casas, F. M. G.; Moura, G. J. B.; Pereira, G. A.; Melo, J. G. M.; Ramos, M. A.; Rodal, M. J. N.; Schiel, N.; Lyra-Neves, R. M.; Alves, R. R. N.; Azevedo-Junior, S. M.; Telino-Junior, W. R.; Severi, W.; The Sci. World. J (2012), doi: 10.1100/2012/205182.

2. Albuquerque, U. P.; Medeiros, P. M.; Almeida, A. L. S.; Monteiro, J. M.; Lins-Neto, E. M. F.; Gomes, J. M.; Santos, J. P.; J. Ethnopharmacol. 2007, 114, 325.

3. Gomes, L. M. C.; Lima-Saraiva, S. R. G.; Andrade, T. M. D.; Silva, J. C.; Diniz, T. C.; Barreto, V. N. S.; Mendes, R. L.; Quintans-Júnior, L. J.; Quintans, J. S. S.; Lima, J. T.; Almeida, J. R. G. S.; J. Young Pharm. 2014, 6, 64 .

4. Gomes, L. M. A.; Andrade, T. M. D.; Silva, J. C.; Lima, J. T.; QuintansJúnior, L. J.; Almeida, J. R. G. S.; Pharmacogn. Res. 2014, 6, 345.

5. Morais, N. R. L.; Oliveira-Neto, F. B.; Melo, A. R.; Bertini, L. M.; Silva, F. F. M.; Alves, L. A.; Rev. Bras. Plantas Med. 2016, 18, 180.

6. Paredes, P. F. M.; Vasconcelos, F. R.; Paim, R. T. T.; Marques, M. M. M.; Morais, S. M.; Lira, S. M.; Braquehais, I. D.; Vieira, I. G. P.; Mendes, F. N. P.; Guedes, M. I. F.; J. Evidence-Based Complementary Altern. Med. 2016, 2016, 1 .
7. Santos, K. A.; Aragão-Filho, O. P.; Aguiar, C. M.; Milinsk, M. C.; Sampaio, S. C.; Palú, F.; Silva, E. S.; Ind. Crops. Prod. 2017, 97, 368.

8. Paula, A. C.; Melo, K. M.; Silva, A. M.; Ferreira, D. A.; Monte, F. J. Q.; Santiago, G. M. P.; Lemos, T. L. G.; Braz-Filho, R.; Militão, G. C. G.; Silva, P. B. N.; Silva, T. G.; Rev. Virtual. Quim. 2016, 8, 231.

9. Endo, Y.; Ohta, T.; Nozoe, S.; Tetrahedron. Lett. 1991, 32, 3083.

10. Lemos, T. L. G.; Silveira, E. R.; Oliveira, M. F.; Braz-Filho, R.; J. Braz. Chem. Soc. 1991, 2, 105.

11. Endo, Y.; Ohta, T.; Nozoe, S.; Tetrahedron. Lett. 1991, 32, 5555.

12. Peixoto-Sobrinho, T. J.; Tavares, E. A.; Castro, V. T. N. A.; VerasFilho, J.; Militão, G. C. G.; Silva, T. G.; Amorim, E. L. C.; Mol. Clin. Pharmacol. 2012, 3, 55.

13. Mensor, L. L.; Menezes, F. S.; Leitão, G. G.; Reis, A. S.; Santos, T. C.; Coube, C. S.; Leitão, S. G.; Phytother. Res. 2001, 15, 127.

14. Falcão, D. Q.; Costa, E. R.; Alviano, D. S.; Alviano, C. S.; Kuster, R. M.; Menezes, F. S.; Braz. J. Pharmacogn. 2006, 16, 73.

15. Oliveira-Júnior, R. G.; Dissertação de Mestrado, Universidade Federal do Vale do São Francisco, Brasil, 2017.

16. Liu, Y.; Wang, H.; Cai, X.; J. Food Sci. Technol. 2015, 52, 2336.

17. Azahar, N. F.; Gani, S. S. A.; Mokhtar, N. F. M.; Chem. Cent. J. 2017 , $11,96$.

18. Bassani, D. C.; Nunes, D. S.; Granato, D.; An. Acad. Bras. Ciênc. 2014, $86,923$.

19. Kraujalis, P.; Venskutonis, P. R.; Ibáñez, E.; Herrero, M.; J. Supercrit. Fluids 2015, 104, 234.

20. Buchner, N.; Krumbein, A.; Rohn, S.; Kroh, L. W.; Rapid Commun. Mass Spectrom. 2006, 20, 3229.

21. Ravber, M.; Pečar, D.; Goršek, A.; Iskra, J.; Knez, Z.; Škerget, M.; J. Agric. Food Chem. 2016, 64, 9196.

22. Al-Dhabi, N. A.; Arasu, M. V.; Park, C. H.; Park, S. U.; EXCLI Journal 2015, 14, 59.

23. La Casa, C.; Villegas, I.; La Lastra, C. A.; Motilva, V.; Calero, M. J. M.; J. Ethnopharmacol. 2000, 71, 45 . 